\title{
Ensino de Química e Deficiência Visual: mapeamento das pesquisas nos CINTEDIs
}

\section{(2014-2020)}

\author{
Teaching of Chemistry and Visual Deficiency: mapping the research in CINTEDI(s) (2014-2020)
}

Enseñanza de la Química y la Discapacidad Visual: mapeo de la investigación en CINTEDIs (20142020)

Recebido: 03/10/2021 | Revisado: 11/10/2021 | Aceito: 13/11/2021 | Publicado: 22/11/2021

\author{
Eleonora Celli Carioca Arenare \\ ORCID: https://orcid.org/0000-0002-6586-6257 \\ Universidade Federal Fluminense, Brasil \\ E-mail: eleonoracelliquimica@gmail.com \\ Gerson de Souza Mól \\ ORCID: https://orcid.org/0000-0002-1964-0513 \\ Universidade de Brasília, Brasil \\ E-mail: gmol@unb.br
}

\begin{abstract}
Resumo
O objetivo desta pesquisa é investigar como está se desencadeando o processo dos registros acadêmicos com relação ao ensinar e aprender Química com relação a Deficiência Visual nos CINTEDIs (2014-2020). Elegemos o mapeamento dos registros de publicações na temática em estudo, explanando de forma discursiva os dados que, em nossa concepção necessitam serem explorados por pesquisas futuras, para isso analisamos especificamente o foco temático Conteúdos Curriculares da disciplina Química. Concluímos que a lacuna de estudo nesta temática é imensa, pois os trabalhos, não dão conta de abordar todos os Conteúdos dos Ensino Médio e nem de Ensino superior, trazendo linhas de tendências, já registradas no contexto brasileiro em outros eventos, com os seguintes conteúdos: Hidrocarbonetos, Tabela Periódica e Misturas, com focos específicos em criação de recursos didáticos inclusivos.
\end{abstract}

Palavras-chave: Ensino de química; Deficiência visual; CINTEDI.

\begin{abstract}
The objective of this research is to investigate how the process of academic records regarding teaching and learning Chemistry in relation to Visual Impairment is being triggered in CINTEDIs (2014-2020). We chose the mapping of publications records on the subject under study, explaining in a discursive way the data that, in our view, need to be explored by future research. For this, we specifically analyzed the thematic focus Curriculum Contents of the Chemistry discipline. We conclude that the study gap in this theme is huge, as the works are not able to address all High School and Higher Education Contents, bringing trend lines, already registered in the Brazilian context in other events, with the following contents: Hydrocarbons, Periodic Table and Blends, with specific focuses on creating inclusive teaching resources.
\end{abstract}

Keywords: Chemistry teaching; Visual impairment; CINTEDI.

\section{Resumen}

El objetivo de esta investigación es indagar cómo se está desencadenando el proceso de expediente académico sobre la enseñanza y el aprendizaje de la Química en relación con la Deficiencia Visual en los CINTEDI (2014-2020). Se optó por el mapeo de registros de publicaciones sobre el tema en estudio, explicando de manera discursiva los datos que, a nuestro juicio, necesitan ser explorados por futuras investigaciones, para ello analizamos específicamente el enfoque temático Contenidos Curriculares de la disciplina Química. Concluimos que la brecha de estudio en este tema es enorme, ya que los trabajos no logran abordar todos los Contenidos de Bachillerato y Educación Superior, trayendo líneas de tendencia, ya registradas en el contexto brasileño en otros eventos, con los siguientes contenidos: Hidrocarburos, Periodic Table and Blends, con enfoques específicos en la creación de recursos didácticos inclusivos.

Palabras clave: Enseñanza de la química; Discapacidad visual; CINTEDI.

\section{Introdução}

Eventos Científicos podem proporcionar momentos que estimulam a Divulgação Científica dentro de contextos regionais ou à nível nacional. Nestes alunos licenciandos tem uma participação mais efetiva, participando geralmente de 
publicações, que maximizam o estímulo a um alavancar profissional estimulado pela importância da pesquisa na trajetória da vida professoral.

Os CINTEDI(s) proporcionam um ambiente para a troca de experiências e ideias com alunos, professores e pesquisadores nacionais e estrangeiros, estimulando ao surgimento e consolidação de grupos de pesquisa, que centralizam pesquisas na área da inclusão envolvendo diversos tipos de disciplinas que englobam a trajetória e a consolidação do processo de ensinar e aprender.

Desde a primeira edição realizada em Campina Grande em Pernambuco em 2014, o Congresso Internacional de Educação Inclusiva se consolidou como o principal evento internacional científico da área de inclusiva aplicada à educação no Brasil. Para pesquisadores e educadores, o Congresso Internacional de Educação Inclusiva (CINTEDI) tem sido um importante ambiente de publicação de resultados, discussão de temas e tendências da área e interação entre grupos que estudam os diversos tópicos que envolvem a área da inclusão.

Conhecer sobre trabalhos relacionando o Ensino de Química a Deficiência Visual, é um objeto de estudo que pode nortear o futuro da Educação Química e da profissão do licenciado em Química. Revisões da literatura podem contribuir para discutir o rumo dos avanços relacionados ao processo como ensinar e proporcionar alunos com Deficiência Visual aprenderem Química.

A questão que surgiu como norteadora deste trabalho é: Que tipos de Conteúdo Curriculares da Grade Curricular de Química em qualquer nível de ensino, estão sendo registradas nos CINTEDIs? Com o intuito de responder essa pergunta, fixamos como objetivo geral: Mapear todas as pesquisas que envolvam Ensino de Química para alunos com Deficiência Visual, num estado temporal de 6 anos de CINTEDIs (2014-2020), apresentados na modalidade de artigo completo.

\section{Fundamentação Teórica}

Muitas pesquisas na área do ensino das Ciências, tem se desenvolvido no intuito de mapear a produção academica em diversas áreas do conhecimento, pois tal conhecimento agrega uma noção do que tem sido estudo, tanto nas Ciências Humanas ( Maçaneiro \& kuhl , 2013; Serva, 2017; Rocha \& Silva, 2021), nas Ciências Exatas (Hayashi et al, 2018; Tavares \& Amaral, 2020; Arenare \& Mól, 2020 ) e nas Ciências da Saúde (Vago, 2013; Fernandes et al, 2020; Ribeiro et al, 2020).

Em se tratando de mapeamento em trabalhos relacionados ao Ensino de Ciências, em diferentes linhas de pesquisa, os pesquisadores ( Delizoicov et al, 2013; Dionor et al, 2013; Tavares \& Amaral, 2020; Scaglioni et al, 2020; Pereira et al, 2021) tem colaborado no sentido de estabelecer uma visão contextual que envolve um contexto histórico, dentro desta perspectiva relacionando o processo de ensino e de aprendizagem de Química ao contexto de alunos com deficiencia visual pesquisadores (Mól et al, 2010; Costa et al, 2015; Reis et al, 2015; Duarte, 2017; Toledo, 2017; Torres et al, 2017; Kopf \& Miranda, 2018; Duarte \& Rossi, 2021), de diferentes regiões brasileiras fizeram registros de seus diferentes tipos de produções academicas (trabalhos de conclusão de curso, eventos,periodicos e dissertações).

Freitas \& Palanch (2015) salientam que "ainda há poucos autores e pesquisadores que se dedicam à realização de amplas pesquisas do tipo Estado da Arte e, menos ainda, aqueles que escrevem e teorizam sobre esse tipo de pesquisa” (p. 785). As pesquisas do estado da arte (estado do conhecimento) são de caráter bibliográfico e buscam mapear uma temática, encontrando as tendências na produção do conhecimento. Nessa vertante, fizemos a construção dessa proposta de pesquisa.

Quando partimos para a Grade Curricular do Ensino da disciplina Química voltada para o ensino médio, temos a seguinte distribuição de Conteúdos Curriculares:

1ANO: As Propriedades da Matéria; A Estrutura Atômica; A Tabela Periódica; As Ligações Químicas; As Funções Inorgânicas; As Reações Químicas; A Estequiometria atômica, molecular e reacional, Cálculo de rendimento e pureza. 
$2^{\circ}$ ANO: Reações Inorgânicas; Estequiometria; Gases; Soluções; Propriedades Coligativas; Termoquímica Química Orgânica

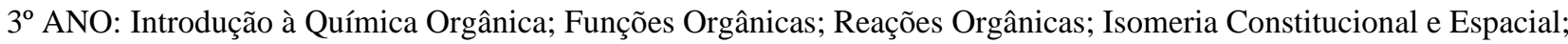
Radiatividade; e Relações da Química com as Tecnologias, a Sociedade e o Meio Ambiente.

Diversificar as aulas, fazendo uma relação entre os Conteúdos Curriculares e a vida cotidiana dos alunos deveria ser uma proposta de todo profissional licenciado em Química, pesquisar, planejar e criar metodologias tanto de ensino quanto de aprendizagem, diversificar as estratégias de ensino e relacionar a vida cotidiana do alunado fazendo uma relação dialógica e cidadã de tais conteúdos, poderia ser uma forma de despertar o interesse e motivar os alunos por tal Ciência, levando-os a compreender a importância relacional entre o porquê de estudar tais conteúdos curriculares na disciplina e suas relações cotidianas com a vida diária em sociedade.

\section{Metodologia}

A pesquisa descrita neste texto foi de natureza bibliográfica, tendo como corpus, os trabalhos publicados nos anais do Congresso Internacional de Educação Inclusiva - CINTEDI no período de 2014 a 2020. Esse evento é bienal e os II e II CINTEDIs aconteceram em parceria com a Jornada Chilena de Educação Inclusiva. A busca pelos textos foi realizada no domínio oficial dos Anais publicados pela Editora Realize, disponibilizados da seguinte forma:

\section{CINTEDI - Anais I CINTEDI | Plataforma Espaço Digital (editorarealize.com.br) \\ II CINTEDI - Anais II CINTEDI | Plataforma Espaço Digital (editorarealize.com.br) \\ III CINTEDI - Anais III CINTEDI | Plataforma Espaço Digital (editorarealize.com.br) \\ IV CINTEDI - Anais IV CINTEDI | Plataforma Espaço Digital (editorarealize.com.br)}

Para a seleção dos trabalhos selecionados neste artigo, ao investigarmos cada um dos anais indicados acima, colocamos para a pesquisa somente trabalhos apresentados como artigo completo, que apresentassem no título do artigo, a palavra "Deficiência Visual" apresentados na modalidade de Comunicação Oral. Assim selecionamos para análise, leitura e estudo os trabalhos específicos com foco no "Ensino de Química para alunos com Deficiência Visual".

O referido evento foi escolhido por ser um evento brasileiro, que dentro da Linha de Educação Inclusiva, em nossa concepção é o início de um olhar a nível nacional, com relação a temática que faz parte de nosso objeto de estudo, o processo de ensino e aprendizagem da disciplina "Química" para alunos com Deficiência Visual, sem desconsiderar que existem outros nos quais também se encontram produções relacionadas. Analisamos a produção acadêmica disponíveis no evento que ocorreram nos seguintes lugares e nos respectivos anos:

Congresso Internacional de Educação Inclusiva - CINTEDI: Todos os CINTEDI(s) aconteceram em Campina Grande - PB, com exceção do IV CINTEDI, realizado em 2020 que ocorreu de forma online.

A metodologia utilizada fundamentou-se na análise de conteúdo (Bardin, 2016), que é um método de tratamento e análise de dados qualitativos em que se procura encontrar convergências e incidências de palavras e frases. A análise de conteúdo segundo a autora é:

Um conjunto de técnicas de análise das comunicações visando obter, por procedimentos sistemáticos e objetivos de descrição do conteúdo das mensagens, indicadores (quantitativos ou não) que permitam a inferência de conhecimentos relativos às condições de produção/recepção (variáveis inferidas) destas mensagens (Bardin, 2011, p. 48). 
Como foco temático de estudo para nossa pesquisa escolhemos os Conteúdos Curriculares que compõem a disciplina Química tanto no Ensino Médio quanto no Ensino Superior. Na seção de resultados e discussões abaixo, apresentamos os resultados que obtidos em nossa investigação.

\section{Resultados e Discussão}

Para termos um panorama da produção registrada no evento, criamos o Quadro 1, com o propósito de quantificar tais publicações e assim verificar o interesse dos profissonais das Licenciaturas em Química, pela pesquisa, estudo e divulgação de seus trabalhos na linha temática objeto de nosso estudo, nesse artigo.

Quadro 1 - Demonstração de dados gerais registrados nos CINTEDI(s)- (2014-2020).

\begin{tabular}{|c|c|c|c|c|c|}
\hline Ano & Evento & $\begin{array}{c}\text { Total de } \\
\text { A. }\end{array}$ & $\begin{array}{c}\text { Total de } \\
\text { C.O. }\end{array}$ & $\begin{array}{c}\text { Total C.O. com } \\
\text { FOCO em D.V. }\end{array}$ & $\begin{array}{c}\text { Total D.V. com } \\
\text { foco em E.Q. }\end{array}$ \\
\hline 2014 & I CINTEDI & 1022 & 685 & 09 & 01 \\
\hline 2016 & II CINTEDI & 872 & 711 & 11 & 00 \\
\hline 2018 & III CINTEDI & 986 & 803 & 16 & 02 \\
\hline 2020 & IV CINTEDI & 320 & 273 & 07 & 01 \\
\hline
\end{tabular}

Legenda: A.= Artigo/C.O.= Comunicação Oral/ D.V.= Deficiência Visual/ E.Q.= Ensino de Química Fonte: ARENARE (2021)

Focamos em analisar somente os trabalhos apresentados na modalidade de Comunicação Oral, conforme o Quadro 1, constatamos que essa forma de apresentação de trabalhos foi a que mais teve número de aceites, entretanto quando se foca especificamente em trabalhos relacionados a Deficiência Visual, é possível se verificar que o índice de interesse dos pesquisadores na temática é inferior a 5\% dos trabalhos publicados em cada um dos CINTEDIs, o que demonstra o pouco interesse dos licenciados pela temática. Focando em nossa área especifica, Ensino de Química para alunos com Deficiência Visual, a quantidade de trabalhos, em cada um dos CINTEDIs é mínima, Além de que em 2016, nenhum trabalho especifico na temática foi registrado.

Na Figura 1, explanamos por meio de um Fluxograma o registro de nossos passos e a quantificação de trabalhos analisados e excluidos para darmos inicio a analise da produção nos CINTEDIs. 
Figura 1 - Fluxograma Descritivo da Produção Academica nos CINTEDI(s) (2014-2016).

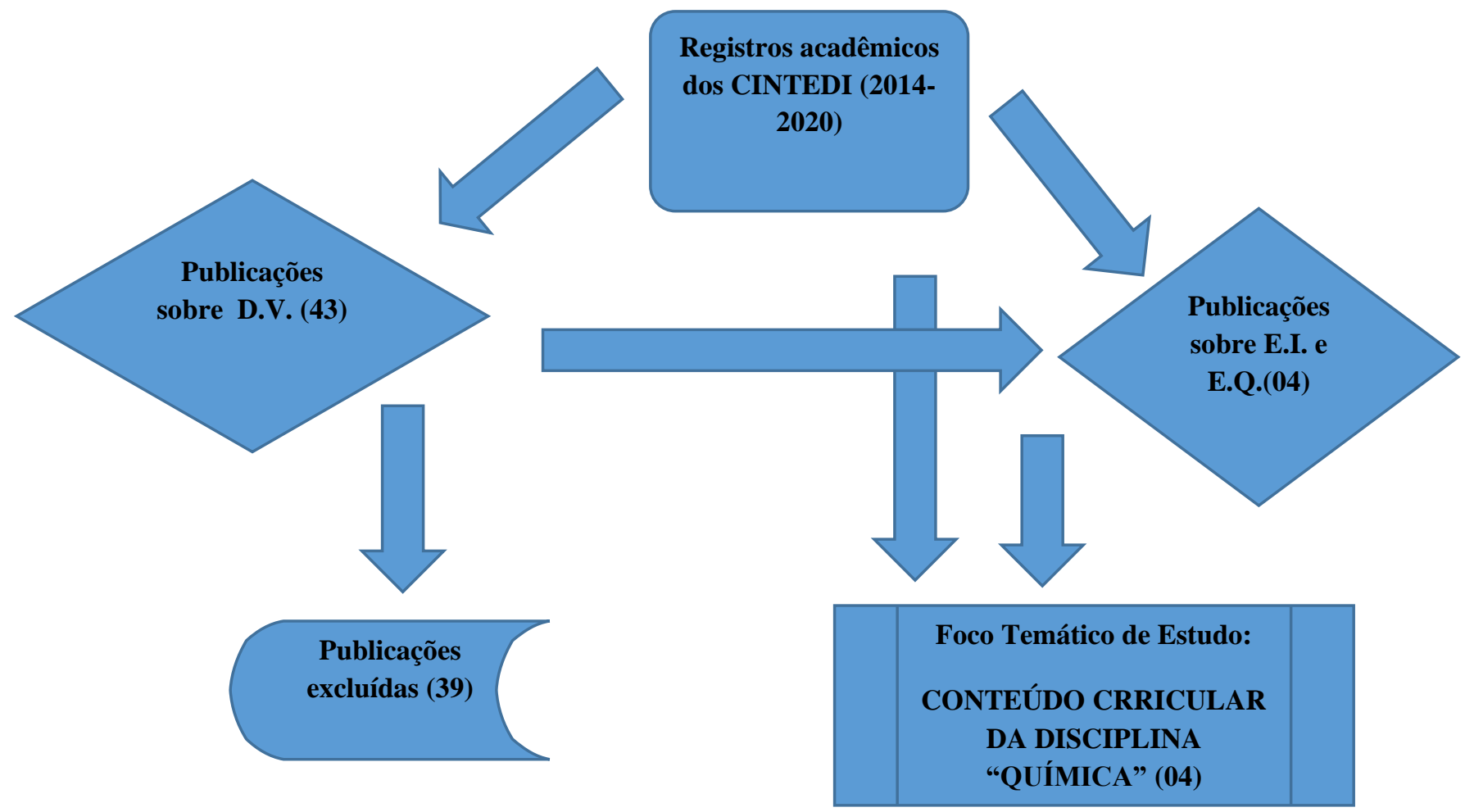

Fonte: ARENARE (2021).

Com o foco de conhecermos melhor sobre cada uma das pesquisas registradas nos CINTEDIs, confeccionamos o Quadro 2, para termos uma ideia da propagação do evento no contexto brasileiro e do foco de estudo dos pesquisadores, como também da origem das IES, que participam de tal evento e assim, darmos inicio a leitura na íntegra dos artigos analisados e desta forma, referenciarmos nossa pesquisa.

Quadro 2 - Registros de dados coletados nos CINTEDI(s) (2014-2016).

\begin{tabular}{|c|c|c|c|c|c|}
\hline Ano & Identificação & Titulo & C.C. & Região & IES \\
\hline 2014 & CINTEDI01 & \begin{tabular}{lr} 
Inclusão de & alunos \\
Deficiência & Visual na \\
elaboração de material didático \\
\multicolumn{3}{c}{ nas aulas de Química }
\end{tabular} & Tabela Periódica & Nordeste & UFPB \\
\hline 2016 & CINTEDI00 & $\begin{array}{l}\text { Não houve publicação com } \\
\text { foco na temática em estudo }\end{array}$ & - & - & - \\
\hline 2018 & CINTEDI02 & $\begin{array}{l}\text { Metodologia para o ensino de } \\
\text { química como ação promotora } \\
\text { da educação inclusiva de } \\
\text { alunos com Deficiência Visual }\end{array}$ & $\begin{array}{l}\text { Fórmulas } \\
\text { estruturais; } \\
\text { Geometria } \\
\text { Molecular; } \\
\text { Tipos de } \\
\text { Ligações; } \\
\text { Hidrocarbonetos } \\
\end{array}$ & Sudeste & IFSP \\
\hline \multirow[t]{2}{*}{2020} & CINTEDI03 & $\begin{array}{l}\text { Proposta de sequência didática } \\
\text { para o ensino de geometria } \\
\text { molecular e polaridade para } \\
\text { turmas inclusivas com alunos } \\
\text { com Deficiência Visual }\end{array}$ & $\begin{array}{l}\text { Geometria } \\
\text { Molecular } \\
\text { Fórmula estrutural } \\
\text { Fórmula Espacial } \\
\text { Fórmula } \\
\text { Geométrica } \\
\end{array}$ & Sudeste & IFSP \\
\hline & CINTEDI04 & $\begin{array}{l}\text { Recurso didático acessível } \\
\text { sobre processos de separação } \\
\text { de misturas para alunos com } \\
\text { Deficiência Visual }\end{array}$ & Misturas & Sudeste & IFRJ \\
\hline
\end{tabular}

Legenda $=$ C.C. $=$ Conteúdos Curriculares. Fonte: ARENARE (2021). 
A presente pesquisa traz uma reflexão sobre o mapeamento de pesquisas (Rodrigues \& Rodrigues, 2014; Disigant et al, 2018; Ferreira et al, 2020; Shimada \& Aricó, 2020; Silva et al, 2020) publicadas nos eventos dos CINTEDIs, conforme evidencia o Quadro 1, com o propósito de mapear o foco dos registros evidenciados a nível nacional, com relação ao desenvolvimento do processo de ensino e aprendizagem de Inclusão de alunos com Deficiência Visual nas aulas de Química.

O Quadro 2 exposto acima, nos permite observar que as regiões Norte, Centro-Oeste e Sul, não participaram com nenhum trabalho, nos eventos realizados nos CINTEDIs, além de nos possibilitar pela tema dos trabalhos, a compreensão de que o foco dado a temática, concentra-se fundamentalmente na linha de metodologias, por meio da criação e confecção de Recursos Didáticos Inclusivos. Somente o trabalho CINTEDI02 faz enfase uma nova metodologia que ajudaria os licenciando em Química na sua atuação profissional, enquanto professsores da disciplina. Com relação aos Conteudos Curriculares explorados, focam mais em Química Organica (50\%) dos trabalhos, destacando-se apenas: Modelos Estruturais da Química Orgânica, Tabela Periódica e Misturas.

Consideramos importante registrar que não existem pesquisas publicadas nos CINTEDI(s) (2014-2020) que explanem registros de trabalhos nos anos finais do Ensino Fundamnetal $\left(9^{\circ}\right.$ ano).

O artigo CINTEDI01 tem como objetivo confeccionar as cartelas e fichas do jogo bingo, tomando como referência o conteúdo da tabela periódica, em que as cartelas seriam elaboradas por grupos de alunos videntes (AV) e alunos com deficiência visual (ADV). Nesse artigo, os autores abordam a ludicidade como estratégia de ensino, numa perspectiva baseada em etapas: 1- Pesquisa Bibliográfica; 2- Atividades em forma grupal; 3- Criação de materiais inclusivos com utilização de materiais de baixo custo, tanto por alunos com Deficiência, quanto por alunos sem Deficiência; .4- Apresentação de seminários; 5- Avaliação das atividades desenvolvidas e realizadas com a turma: 6- Entrevista com alunos com Deficiência Visual e com professores de química.

No registro do CINTEDI01, destaca-se que a forma de contextualizar o conteúdo abordado, trouxe para os alunos o desejo de participar dos jogos, estabelecendo um aprendizado para o alunado, confirmando o que outras pesquisas (Da Cunha, 2012; Moreira et al, 2020; Conde et al, 2021; Nascimento et al, 2021) na área enfatizam também.

Além de trazer uma sintonia de possibilidade da aproximação do aluno com Deficiência Visual dos conceitos científicos, criando a concepção e o entendimento para os alunos de que todos somos seres humanos e que apresentamos diferenças, porém os desafios precisam ser superados de forma harmoniosa com essas diferenças ajudando uns aos outros, convergindo com o que as pesquisas dos estudiosos (Araújo et al, 2020; Silva \& Amaral, 2021) na área também ressaltam.

O CINTEDI 02 vêm explanando a utilização das metodologias como forma de agregar um aprendizado que alcance o aluno com Deficiência Visual, assim como outros pesquisadores (Costa, 2016; Lima, 2017; Toledo et al, 2019; Silva \& Amaral, 2021; Neves et al, 2020) também enfatizam.

Apenas o artigo CINTEDI 02 enfatiza uma abordadagem com o Nível Superior, mas, no contexto de complementação do que se intitula com Criação de uma nova metodologia, que é a Contrução de Modelos tatéis, com utilização de desenhos, esquemas e figuras. Estratégia está já utilizada pelos pesquisadores (Fernandes et al, 2018; Benite \& Benite, 2020) que também salientam a importância de uma metodologia, voltada para a motivação dos alunos com a participação dos alunos sem Deficiência que participam da mesma turma, como forma de integrar e efetuar um ensino e uma aprendizagem, menos exclusiva e mais includente.

Quanto ao CINTEDI 03, tem como objetivo o desenvolvimento de uma sequência e materiais didáticos que promovam a inclusão de discentes com Deficiência Visual. Realizou-se uma análise do Livro Didático Acessível "Ser Protagonista" vigente em 2015- 2017 conforme a temática estabelecida, uma vez que era necessário realizar um estudo sobre como o conteúdo é apresentado no ensino médio para os alunos com Deficiência Visual. A partir daí, fez-se uma pesquisa bibliográfica e elaborou-se um planejamento para a confecção de materiais didáticos de baixo custo, que posteriormente foram 
analisados por uma aluna com Deficiência Visual e uma professora de Atendimento Educacional Especializado (AEE) com o propósito de verificar a efetividade e/ou falhas que poderiam acarretar no aprendizado do aluno.

O artigo CINTEDI 03, colabora com pesquisas realizadas por outros pesquisadores (De Paula et al, 2017; Darim et al, 2021; Maciel, 2016), que enfatizam a importância da associação entre a pesquisa teórica e a pesquisa de atuação de criação de recursos didáticos inclusivos com também autores que estudam a mesma temática e explicam a importância de uma posterior análise dos materiais adaptáveis confeccionados, dentre os quais vale ressaltar as contribuições convergentes dos pesquisadores (Toledo et al, 2019; Franco-Patrocínio et al, 2017; Acris et al, 2019; Almeida et al, 2019; Damasceno et al, 2019) que também trazem suas contribuições enfatizando questões relacionadas a ter um retorno ou de alunos com deficiência visual, de professores de Química que trabalham na lida diária do cotidiano em sala de aula, da comunidade acadêmica ou até mesmo de familiares de alunos com deficiência visual.

O CINTEDI04 versa sobre a produção de um material didático acessível para alunos com deficiência visual na temática dos processos de separação de misturas e dialoga com pesquisadores como Almeida (2017); Sassaki (2007); Cerqueira e Ferreira (1996), que dissertam sobre a importância do material adaptado para pessoas cegas e com baixa visão, e das suas respectivas avaliações. Corroborando com tal aspectos alguns pesquisadores ( Molena, 2018; Matos \& Gomes, 2018; Ruela et al, 2019; Teixeira Júnior \& Souza, 2019 )que também trazem suas contribuições enfatizando questões relacionadas a ter um retorno, com relação a participação de alunos com deficiência visual em sala de aula, por parte dos alunos sem e com deficiência visual e de professores de Química que trabalham na lida diária do cotidiano em sala de aula, da comunidade acadêmica ou até mesmo de familiares de alunos com deficiência visual.

Os trabalhos demonstram uma enorme lacuna, que necessita ser preenchida, pois os conteudos abordados, são de cunho especifico de ensino médio: Hidrocarbonetos, Tabela Periódica e Misturas, com focos específicos em criação de recursos didáticos inclusivos. Assuntos abordados e já enfatizados por grupos de graduandos, mestrandos, doutorandos, pesquisadores, estudiosos e professores da área de Ensino de Química.

\section{Considerações Finais}

É inegável que a condução o processo de ensino e aprendizagem de Quimica para qualquer nível de ensino, sofre intervenções positivas ou negativas do profissional professor de Química, até porque esse é um ator protagonista no cenário contextual da sala de aula, em qualquer contexto regional brasileiro.

A orientação dada na trajetória do processo de ensino do profissional professor de Química, deveria desencadear uma multi interface de atividades adaptativas, para que a aprendizagem alcance o processo cognitivo de alunos com Deficiência Visual e alunos sem qualquer tipos de deficiência, gerando estratégias de metodologias que influenciem a absorção do que a prática diária de tal profissional exige, um olhar não especifico somente nos conteúdos mas, também na lida diária dentro da sala, na absorção da aprendizagem pelos aunos que cruzam sua trajetória e também influenciar a motivição gerada na cognição dos alunos, fato este complexo, gerado apartir da diversificação das aulas aplicadas diariamente.

Nossa pesquisa demonstra o quanto ainda existe a necessidade de criação de metodologias que envolvam os Conteudos Curriculares de Química, em diferentes modalidades de ensino, porque a cognição de um aluno com Deficiencia Visual não sofre interferências de sua limitação, ou seja, alunos com Deficiência Visual tem a memória perfeita no sentido da assimilação da aprendizagem de Conceitos Cientificos, fato este, muitas vezes ignorado por professores que lecionam a disciplina de Quimica.

O que subsidia um questionamento que dar margem para a seguinte questão norteadora: Porque trabalhos relacionados a Deficiência Visual registrados por meio de publicações, sempre enfatizam os mesmos Conteúdos Curriculares na disciplina Química? Tendo em vista que, nos enquanto professores necessitamos sermos autores das práticas pedagógicas e 
metodológicas que ensinamos em nossas salas de aula. Seria isto, uma deficiência em nossa formação inicial ou um processo de acomodação que se estabelece cotidianamente pela falta de autonomia professoral na busca de uma formação continuada mais interessada, atualiza e diversificada? Não é nossa pretensão responder por meio dessa pesquisa tal questionamento, mas, instigar nossos colegas de profissão para essa questão tão importante para o processo de melhoria de nossas aulas, enquanto profissionais da área.

Como sugestão para futuros trabalhos, é necessário a criação de novas formas de abordar os Conteúdos Curriculares de Química, até então já divulgados neste evento e em outros eventos nacionais, caso isto não seja concretizado, estará se fazendo uma repetição de dados, manipulando-se somente o cenário contextual onde os mesmos têm sido realizado, o que exige do profissional professor um certo grau de autonomia na questão da sua prática professoral e as pesquisas realizadas na temática estudada. Enfatizamos que a leitura sobre questões envolvendo tal temática é essencial para que isto aconteça.

\section{Referências}

Acris, D., Barros, A. S., Dos Santos, B. O., Da Silva, D. R., Wadick, E. V., Procópio Junior, P. S., Brandão, E. G., \& De Lemos, R. G. (2019). Proposta de prática experimental para alunos com deficiência visual no contexto das limitações e possibilidades no interior do Amazonas. $17^{\circ}$ Simpósio Brasileiro de Educação Química - $17^{\circ}$ SIMPEQUI, Porto Alegre, RS, Brasil.

Almeida, M. G. S. Ver além do visível: a imagem fora dos olhos. (2017). Tese de doutorado - Programa de Pós-Graduação em Literatura, Cultura e Contemporaneidade. Pontifícia Universidade Católica do Rio de Janeiro, Rio de Janeiro.

Almeida, J. P. C., Saito, E. K. N., Martins, T. R., \& Kato, O. M. (2019). Confecção de recursos didáticos para o ensino de pessoas Cegas por graduandos de licenciaturas de Química e Geografia. Anais do VI Congresso Nacional de Educação - VI CONEDU, Editora Realize, Campina Grande, PB, Brasil.

Araujo, S. A., Rocha, M. E., \& Santos, M. O. (2020). Teaching chemistry to visually impaired students: Challenges and possibilities. Brazilian Journal of Development, v. 6, p. 57156-57177.https://www.brazilianjournals.com/index.php/BRJD/article/view/14826

Arenare, E. C. C., Mól, G. S. Inclusive education and visual disability: mapping chemistry teaching in natioonal Science teaching research meetings (ENPECs - 1997-2017). Research, Society and Development, 9(5), e22953047, 10.33448/rsd-v9i5.3047. https://rsdjournal.org/index.php/rsd/article/view/3047

Bardin, L. (2016). Análise de Conteúdo. Edições 70.

Benite, A. M. C., Benite, C. R. M. (2020). Ensino de Química para alunos com deficiência visual: Estudos sobre a formação de modelos mentais de compostos orgânicos. Benjamin Constant, 1(60), 6-28. http://revista.ibc.gov.br/index.php/BC/article view/758

Cerqueira, J. B., \& Ferreira, E. M. B., (1996). Os recursos didáticos na educação especial. Revista Benjamin Constant, Rio de Janeiro, 5, 15-20. http://www.ibc.gov.br/images/conteudo/revistas/benjamin_constant/2000/edicao-15-abril/Nossos_Meios_RBC_RevAbr2000_ARTIGO3.pdf

Conde, I. B., Jacinto Junior, S. G., Silva, M. A. M., \& Veras, K. M. (2021). Percepções de professores de Química no período da pandemia de Covid-19 sobre o uso de jogos virtuais no ensino remoto. Research, Society and Development, 10(10), e550101019070. 10.33448/rsd-v10i10.19070. https://rsdjournal.org/index.php/rsd/article/view/19070

Costa, E. L. da. (2016). A formação de conceitos científicos para sujeitos com deficiência visual: sequência fedathi como aporte metodológico no Ensino de Química. (Dissertação de mestrado -Programa de pós-graduação em Educação Brasileira, Universidade Federal do Ceará, Fortaleza, CE, Brasil).

Costa, F. R. S., Paula, T. E., \& Camargo, S. (2015). Análise das publicações dos encontros nacionais do Ensino de Química (ENEQ) acerca da elaboração de materiais didáticos para alunos com deficiência visual. Anais do VI Encontro Nacional de Pesquisa em Educação em Ciências - Associação Brasileira de Pesquisa em Educação em Ciências - Atas do X ENPEC - Universidade Federal de Santa Catarina.

Da Cunha, M. B. (2012). Jogos no ensino de química: considerações teóricas para sua utilização em sala de aula. Química Nova na Escola, 34(2), 92-98. qnesc.sbq.org.br/online/qnesc34_2/07-PE-53-11.pdf

Damasceno, R. I. O., Andrade, P. L. M., Freitas, L. S., \& Forte, C. M. S. (2019). Jogo tátil tridimensional inclusivo para alunos com Deficiência visual e Normovisuais como recurso lúdico de compreensão da Tabela Periódica. Anais do VI Congresso Nacional de Educação - VI CONEDU, Editora Realize, Campina Grande, PB, Brasil.

Darim, 1., Guridi, V., \& Amado, B. (2021). A multissensorialidade nos recursos didáticos planejados para o Ensino de Ciências orientado a estudantes com deficiência visual: Uma revisão da literatura. Revista Educação Especial (UFSM), 34(7), 1-28. Doi: https://doi.org/10.5902/1984686x48289

Delizoicov D., Slongo, I. I. P., \& Lorenzetti, L., (2013). Um panorama da pesquisa em educação em ciências desenvolvida no Brasil de 1997 a 2005. Revista Electrónica de Enseñanza de las Ciências, 12(3), 459-480. http://reec.uvigo.es/volumenes/volumen12/REEC_12_3_5_ex718.pdf

De Paula, T. E., Guimarães, O. M., \& Silva, C. S. da. (2017). Necessidades formativas de professores de Química para a inclusão de alunos com deficiência visual. Revista brasileira de Pesquisa em Educação em Ciências, 17(3), 853-881. https://doi.org/10.28976/1984-2686rbpec2017173853

Dionor, G. A., Ferreira, R. L., \& Martins, L. (2013). Análise da temática educação em saúde em atas de evento sobre Educação em Ciências. Candombá. 9, p. 22-34. http://web.unijorge.edu.br/sites/candomba/pdf/artigos/2013/a3.pdf 
Disigant, I., Santos, J. G., \& Aricó, E. M. (2018). Metodologia para o Ensino de Química como ação promotora da educação inclusiva de alunos com deficiência visual. Anais do III Congresso Internacional de Educação Inclusiva (III CINTEDI) - Editora Realize, Campina Grande, PB, Brasil.

Duarte, C. C. C. (2017). Ensino de Química para pessoas com deficiência visual: mapeamento e investigação de produções e aplicações no Brasil. Anais do VII Seminário interno do Programa de pós-graduação Multiunidades em Ensino de Ciências e Matemática - Campinas.

Duarte, C. C. C., \& Rossi, A. V. (2021). Ensino de Química para pessoas com deficiência visual: Mapeamento e investigação de produções no Brasil. REEC. Revista Electrónica de Enseñanza de las Ciencias, 20, 396-421. http://reec.uvigo.es/volumenes/volumen20/REEC_20_3_4_ex1867_628.pdf

Ferreira, A. S., Souza, L. C. A. B., Gomes, M. N., \& Barth, A. (2020). A evolução dos estudos sobre questões sociocientíficas: caracterização dos trabalhos apresentados em eventos brasileiros da área de Ensino de Ciências. South American Development Society Journal, 6(18), 257-272. https://www.sadsj.org/index.php/revista/article/view/359

Fernandes, J. M., Franco-Patrocínio, S. O., \& Freitas-Reis, I. (2018). Possibilidades para o fazer docente junto ao aprendiz cego em aulas de Química: uma interface com a história da Tabela Periódica. História da Ciência e Ensino: Construindo Interfaces, 18, 181-199. http://dx.doi.org/10.23925/21782911.2018v18p181-199

Fernandes, F., Pereira, O., \& da Silva, L., (2020). Um Panorama das Pesquisas Científicas focado no ENCompIF: Um Mapeamento Sistemático. Anais do Encontro Nacional DE computação DOS Institutos Federais (ENCOMPIF), Porto Alegre, RS, Brasil.

Franco-Patrocínio, S., Fernandes, J. M., \& Freitas-Reis, I. (2017). Um modelo tátil da Tabela Periódica: O Ensino de Química para alunos cegos num contexto inclusivo. XI Encontro Nacional de Pesquisa em Educação em Ciências - Associação Brasileira de Pesquisa em Educação em Ciências - Atas do XI ENPEC Universidade Federal de Santa Catarina/ Florianópolis, Brasil.

Freitas, A. V., \& Palanch, W. B. de L. (2015). Estado da Arte Como Metodologia de Trabalho Científico na Área de Educação Matemática: Possibilidades e Limitações. Perspectivas da Educação Matemática, 8(18), 784-802. https://periodicos.ufms.br/index.php/pedmat/article view/867/983

Hayashi, M., Maroldi, A., Camargo, J., Godói, F., \& Hayashi, C. (2018). Gênero nos estudos bibliométricos apresentados nos ENANCIBs (19942016). Revista ACB, 23(1), 54-68. https://revista.acbsc.org.br/racb/article/view/1396

kopf, A. C. S., \& Miranda, A. C. D. (2018). Mapeamento das publicações em Química na Revista Brasileira de Pesquisa em Educação em Ciências (RBPEC) de 2014 - 2018. Revista Educar Mais, 2(1). https://doi.org/10.15536/reducarmais.2.2018.\%p.1236

Layter, M. B., Fioresi, C. A., Cunha, M. B. , \& Silva, V. M. A avaliação nos trabalhos sobre jogos didáticos apresentados no Encontro Nacional de Ensino de Química. III Congresso paranaense de Educação Química - Universidade Estadual de Ponta Grossa.

Lima, B. T. da S. (2017). Proposta de química orgânica para alunos com deficiência visual: desenhando prática pedagógica inclusiva. Dissertação (Programa de pós-graduação em Ensino de Ciências e Educação Matemática - PPGECEM, Universidade Estadual da Campina Grande, PB, Brasil).

Maciel, A. P., Batista Filho, A., \& Prazeres, G. M. P. (2016). Equipamentos alternativos para o Ensino de Química para alunos com deficiência visual. Revista Docência do Ensino Superior, Belo Horizonte, 6(2), 153-176. 10.35699/2237-5864.2016.2106. https://periodicos.ufmg.br/index.php/rdes/article/view/2106

Maçaneiro, M. B., \& Kuhl, M. R. (2013). Estado da arte e o rumo do conhecimento científico em secretariado executivo: mapeamento e análise de áreas de pesquisa. Revista de Gestão e Secretariado, 4(3), 157-188, 2013. https://www.revistagesec.org.br/secretariado/article/view/274

Matos, F. I., \& Gomes, L. C. (2018). Reflexões de licenciandos sobre o enino de Química a alunos com deficiência visual. Revista Brasileira de Educação Básica, 3, 1-6. http://pensaraeducacao.com.br/rbeducacaobasica/wp-content/uploads/sites/5/2018/06/05 -Fernanda-Alves-REFLEXÕES-DELICENCIANDOS.pdf

Mól, G. S., Raposo, P. N., Santos, G. A., Neto, J. D., \& Brito, A. G. (2010). A inclusão de alunos com deficiência visual como tema em dissertações e teses nos programas de pós-graduação da área de Ensino de Ciências e Matemática da CAPES. Anais do XV Encontro Nacional de Ensino de Química (XV ENEQ), Brasília, DF.

Molena, J. C. (2018). Ensino de Química para alunos com deficiência visual: investigando a percepção de professores sobre o processo de conceitualização. Dissertação (Mestrado em Educação em Ciências e Matemática) - Universidade Federal de São Carlos, Araras, Brasil.

Moreira, L. M., Araújo Neto, W. N., \& Pinto, G. A. (2020). Ensino de Química e Jogos Teatrais: uma pesquisa sobre a intencionalidade dos gestos. Revista Apeduc Journal, 1, 73-88. https://apeducrevista.utad.pt/index.php/apeduc/article /view/103/31

Nascimento, P. H. B., Santos, k. L., Aguiar, L. F., Alcantara, A. C., Silva, M. G., Silva, R. S. T., Jacob, I. T. T., \& Silva, P. R. (2021). Evaluation of learning in the teaching of Chemistry in the final series of elementary school, through the production of didactic games. Brazilian Journal of Development, 7, 7449574499. https://www.brazilianjournals.com/index.php/BRJD/article/view/33505/0

Neves, D. C. F. N., Sousa, J. K. C., Ferreira, M. S., \& Souza, B. F. de. (2020). Série Educar - Educação Especial e Inclusiva. (Org.). Leitor acessível: uma proposta de dispositivo para medidas de massa em aulas práticas de química para pessoas com deficiência visual. 1ed.: Editora Poisson.

Pereira, A., Dorneles, P., \& Balladares, A. (2021). Feiras de Ciências: Um levantamento bibliográfico sobre processos de escrita e avaliação de resumo. Revista Insignare Scientia - RIS, 4(3), 582-595. https://periodicos.uffs.edu.br/index.php/RIS/article/view/12144/7838

Reis, L. S. A., Araújo, A. C. B., \& Ribeiro, K. P. (2015). O desenvolvimento web no processo de ensino e aprendizado de Química para deficientes visuais. Simpósio hipertexto e tecnologias da Educação, Recife, PE, Brasil.

Ribeiro, A. P., Oliveira, G. L., Silva, L. S., \& Souza, E. R. (2020). Saúde e segurança de profissionais de saúde no atendimento a pacientes no contexto da pandemia de Covid-19: revisão de literatura. Revista Brasileira Saúde Ocupacional. 45, e25-e25. https://doi.org/10.1590/2317-6369000013920 
Rocha, C. S. da. (2016). O estado do conhecimento sobre o ensino de história na eja: um estudo a partir dos anais dos simpósios da associação nacional de história (ANPUH-BRASIL) 1961-2015. Dissertação. Programa de pós-graduação em Educação. Universidade Federal da Fronteira Sul - UFFS. https://rd.uffs.edu.br/handle/prefix/699

Rocha, E. A., \& Silva, A. J. N. (2021). Conjecturas a partir de um mapeamento: O ensino de álgebra nos anos finais do ensino fundamental. Caminhos da Educação Matemática em Revista (on-line), 11, 167-189. https://aplicacoes.ifs.edu.br/periodicos/caminhos_da_educacao_matematica/article/view/902

Rodrigues, E. G. S. \& Rodrigues, I. A. (2014). Inclusão de alunos com deficiência visual na elaboração de material didático nas aulas de Química. Anais do CINTEDI - Congresso Internacional de Educação e Inclusão - Práticas pedagógicas, Direitos Humanos e Interculturalidade. Editora Realize. Campina Grande, PB, Brasil.

Ruela, B. A, Morais, A. A. A. M., Oliveira, M. S. G., França, F. A., \& Benite, C. R. M. (2019). Condicionador: Experimento para discussão sobre aminas e sal de amônio quaternário envolvendo alunos com Deficiência visual . Anais do $17^{\circ}$ Simpósio Brasileiro de Educação Química - SIMPEQUI, Porto Alegre, RS, Brasil.

Sassaki, R. K. (2007). Nada sobre nós, sem nós: Da integração à inclusão - Parte 2. Revista Nacional de Reabilitação, 58, 20-30. https://www.sinprodf.org.br/wp-content/uploads/2012/01/nada-sobre-nÓs-sem-nÓs2.pdf

Scaglioni, C. G., Pereira, B. A. I., Rodrigues, T. de M., Leite Filho, I., \& Dorneles, P. F. T. (2020). Estudo de teses e dissertações nacionais sobre feiras de Ciências: mapeamento dos elementos que envolvem uma feira de ciências e suas interligações. Revista Educar Mais, 4(3), 738-755. https://doi.org/10.15536/reducarmais.4.2020.2012

Serva, M. (2017). Epistemologia da administração no Brasil: o estado da arte. Cadernos ebape.br (FGV), 15, 740-750. https://bibliotecadigital.fgv.br/ojs/index.php/cadernosebape/article/view/73209

Shimada, M. S., \& Arico, E. M. (2018). Proposta de sequência didática para o ensino de Geometria molecular e polaridade para turmas inclusivas com alunos com deficiência visual. III Congresso Internacional de Educação Inclusiva (III CINTEDI), Campina Grande, PB, Brasil.

Silva, A. C., Cid, T. P., Rocha, A. C. S., Penco, V. S. N., \& Estevão, A. P. S. S. (2020). Recurso didático acessível sobre processos de separação de misturas para alunos com Deficiência Visual. Anais do IV Congresso Internacional de Educação Inclusiva (IV CINTEDI) e V Jornada Chilena Brasileira de Educação Inclusiva, Campo Grande. Editora Realize. Campina Grande, PB, Brasil.

Silva, R. S., \& Amaral, C. L. C. (2021). As contribuições da defectologia e da teoria histórico-cultural no Ensino de Química para o deficiente visual: Um estado da arte em teses e dissertações (2000-2019). Revista Communitas, 5, 1-19. https://periodicos.ufac.br/index.php/COMMUNITAS/article/view/4699

Tavares, A. P., \& Amaral, C. L. C. (2020). A utilização de histórias e quadrinhos no ensino de química: um mapeamento da produção científica nos ENPECs (PERÍODO 2011-2019). Anais do CIET:EnPED:2020 - (Congresso Internacional de Educação e Tecnologias Encontro de Pesquisadores em Educação a Distância), São Carlos, ago. 2020. ISSN 2316-8722. https://cietenped.ufscar.br/submissao/index.php/2020/article/view/1102

Teixeira Júnior, J. G., \& Souza, N. C. (2019). Análise das concepções de formadores de professores de Química acerca da inclusão de alunos com deficiência no ensino superior. Ensino Em Re-Vista, 26(2), 437-456. https://doi.org/10.14393/ER-v26n2a2019-7

Toledo, J. B. (2017). Ensino de Química para pessoas com deficiência visual: um estudo por meio da revisão sistemática. Tese de Doutorado, Programa de pós-graduação em Química, Universidade Federal de São Carlos, UFSCAR., São Paulo, SP, Brasil.

Toledo, K. C., Santos, B. M., \& Rizzatti, I. M. (2019). O uso da impressora 3D na construção de geometrias moleculares como uma proposta didática no Ensino de Química, adaptado para pessoas com deficiência visual. VI Congresso Nacional de Educação (VI CONEDU), Fortaleza, CE, Editora Realize, Brasil.

Torres, J. C., Ferreira Júnior, J. L., \& Pessôa, P. A. P. (2017). Oficina Interativa: produção de recursos alternativos para ensino de química aos alunos cegos ou de baixa visão. IV Congresso Nacional de Educação (IV CONEDU), João Pessoa, PB, Editora Realize, Brasil.

Vago, T. M. (2013). Escrevendo histórias de educação física, de esportes e de lazer: uma apreciação da produção a partir do CEMEF/UFMG. Organizando arquivos, produzindo nexos: a experiência de um centro de memória. Belo Horizonte, MG, Brasil. Editora: Fino Traço. 